\title{
Datenschutz-Kodex für Geodatendienste und Gesetzentwurf des BMI zur „roten Linie" sind unzureichend!
}

Unter der Überschrift „Mehr Datenschutz für Internet-Nutzer" hat der Bundesinnenminister einen Gesetzentwurf zur Veröffentlichung von personenbezogenen Daten im Internet vorgestellt. Die Bundesregierung will demnach eine "gezielte Verbreitung von Persönlichkeitsprofilen“ nur dann erlauben, wenn die Betroffenen dem zugestimmt haben oder ein „klar überwiegendes Interesse an der Veröffentlichung“ bestehe. Die kommerzielle Veröffentlichung von Daten im Netz solle verboten sein, wenn sich dadurch ein „umfangreiches Persönlichkeits- oder Bewegungsprofil des Betroffenen ergeben“" könne oder der Betroffene „in ehrverletzender Weise " beschrieben oder abgebildet werde.

Dieser Gesetzentwurf muss aus der Sicht des Datenschutzes als verfehlt abgelehnt werden. Unter dem Vorwand, eine „rote Linie“ ziehen zu wollen, wird allen denkbaren Veröffentlichungen von personenbezogenen Informationen im Internet bescheinigt, dass sie grundsätzlich zulässig sind: Unzulässig sollen sie nämlich nur sein, wenn die „rote Linie“ überschritten wird, die nach Aussage des Bundesinnenministers bewusst , hoch “ angesetzt worden sei, da das Internet als öffentlicher Raum „grundsätzlich frei von staatlichen Restriktionen sein sollte“. Die Bundesregierung setze ergänzend auf eine freiwillige Selbstverpflichtung der Industrie.

Woher allerdings die Motivation der Industrie kommen soll, solche Selbstverpflichtungen zu schaffen, wenn das Gesetz einen umfassenden Persilschein für ihre Internet-Veröffentlichungen schafft, bleibt das Geheimnis des Innenministers.

Inhaltlich bringt der Gesetzentwurf für die Betroffenen keine im Vergleich zur bestehenden Rechtslage neuen Ansprüche: Bereits jetzt haben Bürger, deren Persönlichkeitsrechte durch Internet-Veröffentlichungen beeinträchtigt werden, einen Anspruch auf Unterlassung sowie auf Schmerzensgeld und sonstige Wiedergutmachungsmaßnahmen (Ansprü- che aus $\$ \$ 823$ Abs. 2, 826, 1004 Bürgerliches Gesetzbuch). Aus meiner Sicht ist es demgegenüber erforderlich, dass der Bürger vom Gesetzgeber als Herr seiner Daten ernst genommen wird. Seine Rechte im Internet müssen umfassend gesetzlich geschützt werden; „Rote Linien“, durch die alle möglichen Persönlichkeitsrechtsverletzungen unterhalb der Schwelle des „schweren Eingriffs“ legitimiert werden, schaden eher, als dass sie nützen.

Wie defizitär Selbstverpflichtungen sind, zeigt der vom BITKOM vorgelegte Entwurf eines Datenschutz-Kodex zu Google Street View und ähnlichen Diensten. Wie der hamburgische und der nordrhein-westfälische Kollege kritisiere auch ich den Ansatz, den der Bitkom mit dem Kodex für Geodatendienste verfolgt: Weder sind die Unternehmen, die der Selbstverpflichtungserklärung nicht beitreten, künftig verpflichtet, die Vorgaben einzuhalten, noch lassen sich Verstöße gegen den Kodex durch eine selbständige Datenaufsicht durchsetzen und sanktionieren.

Außerdem muss der Bürger die Veröffentlichung seines Lebensumfelds im Internet verhindern können, bevor die Bilder im Netz stehen; eine Widerspruchsmöglichkeit, die zur Voraussetzung hat, dass das Kind in den Brunnen gefallen ist, d.h. dass die zu löschenden oder zu schwärzenden Bilder klar erkennbar im Internet abrufbar sind, greift deutlich $\mathrm{zu}$ kurz. Dies aber ist wesentlicher Teil des gegenwärtigen Konzeptes des vorgelegten Datenschutzkodex.

Auch die vorgesehene Löschung der Rohdaten ist nicht datenschutzgerecht: Eine Frist von einem Jahr für Bilder von $\mathrm{Ge}$ sichtern und KFZ-Kennzeichen ist deutlich zu lang.

Aus meiner Sicht müssen schwarze Schafe unter den Unternehmen, die sich nicht an den Willen der Bürger halten, wirksame Sanktionen zu befürchten haben. Diesem Anliegen wird der Kodex nicht gerecht. Wer den Datenschutz ernst nimmt, sollte stattdessen den bereits vor
Wochen von der rheinland-pfälzischen Landesregierung wesentlich mitgestalteten und im Bundesrat von allen Bundesländern gebilligten Gesetzentwurf zu diesen Internet-Angeboten weiter verfolgen (BT-Drs. 17/2765). Diese gesetzliche Regelung kann nicht durch einen von den Unternehmen selbst formulierten Kodex im Sinne eines „soft law“ ersetzt werden. Aus datenschutzrechtlicher Sicht unverzichtbar sind daher die im Bundesratsentwurf enthaltenen Regelungen:

- Gesichter und amtliche Kennzeichen sind unkenntlich zu machen.

- Die Betroffenen sind frühzeitig durch öffentliche Bekanntmachungen der Datenerfasser über deren Vorhaben zu unterrichten.

- Widerspruchsmöglichkeiten müssen bereits vor der Veröffentlichung im Internet eingeräumt werden.

- Aufnahmen, die unter „Überwindung blickschützender Vorrichtungen“ erstellt werden, bedürfen grundsätzlich der Einwilligung; damit wird auch ein Schutz vor Aufnahmen aus der Vogelperspektive erreicht.

- Die von Widersprüchen betroffenen Aufnahmen sind auch im Rohdatenbestand (nicht nur im Bestand der veröffentlichten Aufnahmen) frühzeitig zu löschen.

- Empfindliche Bußgeldandrohungen müssen die Umsetzung dieser Anforderungen sichern.

Die Bundesregierung hat auch in ihrer dazu abgegebenen Stellungnahme (Anlage 2 zur BT-Drs. 17/2765) deutlich gemacht, dass sie ein größeres Interesse an den mit Internetveröffentlichungen verbundenen Geschäftsinteressen als am Schutz der Betroffenenrechte hat. Dies bedauere ich sehr.

Edgar Wagner

Landesbeauftragter für den Datenschutz Rheinland-Pfalz 